\title{
Possible involvement of stem-like populations with elevated ALDH1 in sarcomas for chemotherapeutic drug resistance
}

\author{
KANYA HONOKI ${ }^{1}$, HIROMASA FUJII ${ }^{1}$, ATSUSHI KUBO ${ }^{2}$, AKIRA KIDO ${ }^{1}$, \\ TOSHIO MORI $^{3}$, YASUHITO TANAKA ${ }^{1}$ and TOSHIFUMI TSUJIUCHI ${ }^{4}$ \\ Departments of ${ }^{1}$ Orthopedic Surgery and ${ }^{2} 1$ st Internal Medicine, and ${ }^{3}$ RI Center, Nara Medical University; \\ ${ }^{4}$ Department of Life Science, Faculty of Science and Technology, Kinki University, Japan
}

Received February 5, 2010; Accepted March 26, 2010

DOI: $10.3892 /$ or_00000885

\begin{abstract}
Elevated aldehyde dehydrogenase 1 (ALDH1) has been proposed as one of the possible candidates for a stem cell maker that can be used for the isolation of cancer stem cells from cancers such as leukemia and breast cancer. In the current study, we found that subpopulations with elevated ALDH1 were present in the human sarcoma cell lines, MG63 (osteosarcoma) and HT1080 (fibrosarcoma), using Aldefluor assay. Both ALDH1 positive and negative cell populations were isolated from the MG63 cell line, by cell-sorting using FACSAria. Both subpopulations had a comparable ability to differentiate similarly to the parental MG63, under normal monolayer culture condition. Subpopulations with the ability to form spheres in anchorage-independent, serum-starved conditions showed increased ALDH1 mRNA expression in addition to strong mRNA expression of the stem cell-related genes, such as Nanog, Oct3/4, Stat3 and Sox2, and possessed ability for self-renewal with secondary sphere formation. Sarcosphere cells from the MG63 cell line showed strong chemo-resistance against both doxorubicin and cisplatin compared with monolayer, adherent cells. In conclusion, sphere-forming cells with elevated ALDH1 are a possible candidate for sarcoma stem cells, possessing strong chemoresistant capacities. Although ALDH1 elevation was not sufficient for representing sarcoma stem cells, the efficient detoxification could contribute to the chemo-resistant properties of the stem-like sphere cells form human sarcoma.
\end{abstract}

\section{Introduction}

Stem cells were proposed as the cell of origin of cancers a number of years ago (1). This hypothesis was further supported by the cancer stem cell model which depicts the

Correspondence to: Dr Kanya Honoki, Department of Orthopedic Surgery, Nara Medical University, 840 Shijo-cho, Kashihara, Nara 634-8521, Japan

E-mail: kahonoki@naramed-u.ac.jp

Key words: sarcoma, cancer stem cell, aldehyde dehydrogenase 1, drug resistance presence of rare side-population within the tumor that can initiate and generate cancerous tissues (2). Cancer stem cells share similar properties with normal stem cells, including the ability of self-renew, resulting in differentiation and tissue generation (3). The presence of these cells was first described in acute myeloid leukemia (4), and subsequently extended to a variety of solid tumors, including cancers of breast, brain, prostate, skin, colon, pancreas, and head and neck (5-11). While several surface markers have been proposed in leukemia and certain epithelial cancers $(5,12)$, possible cancer stem cell markers, which are ubiquitously expressed across multiple cancers, representing conserved stem and progenitor cell functions, have not yet been identified. Aldehyde dehydrogenase 1 (ALDH1) has been proposed as a possible marker fitting this hypothesis. ALDH1 is a cytosolic detoxifying enzyme responsible for the oxidation of intracellular aldehydes $(13,14)$. It is highly expressed in hematopoietic progenitors, where it is linked to retinoid metabolism with a role in early differentiation of stem cells, through the oxidization of retinol to retinoic acid (15). It is also believed to be responsible for the resistance to the alkylating agent of hematopoietic stem cells (16). Furthermore, Storms et al demonstrated that primitive hemtopoietic progenitors could be purified with ALDH activity suggesting, that ALDH1 might be a reliable marker for the isolation of stem cells (17). Increased ALDH1 activity has also been found in stem cell populations in acute myeloid leukemia and breast cancers, as well as their normal counterparts; mammary and hematopoietic stem cells, and has been found to be correlated with drug resistance and poor prognosis of patients (18-20). Thus, ALDH1 has been proposed as a common marker for both normal and malignant stem cells.

In the current study, we demonstrate the presence of increased ALDH1 activity in populations of human sarcoma cell lines, using Aldefluor ${ }^{\circledR}$ assay system (21). We also characterized stem cell properties through the formation of spheres in anchorage-independent, serum-starved conditions, to test whether ALDH1 could be a potential marker for sarcoma stem cells. The results showed that populations with elevated ALDH1 activity are present in human sarcoma cell lines and sphere forming populations with stem cell properties showed strong ALDH1 expression, suggesting that the populations with high ALDH1 activity contains the stem-like cell populations, i.e. sarcoma stem cells. 


\section{Materials and methods}

Cell culture. Human MG63 osteosarcoma and HT1080 fibrosarcoma cell lines were used in this study. Both cell lines were obtained from the American Type Culture Collection (ATCC, USA) and maintained in Dulbecco's minimum essential medium (DMEM) supplemented with $10 \%$ fetal bovine serum (FBS) at $37^{\circ} \mathrm{C}$ in a $5 \% \mathrm{CO}_{2}$ atmosphere.

Aldefluor assay and separation of the ALDH subpopulations by FACS. The Aldefluor ${ }^{\circledR}$ (StemCell Technologies, USA) was used to isolate cell populations with high ALDH enzymatic activity. Cells were labeled with Aldefluor reagent, as per the manufacturer's instructions. Briefly, cultures of MG63 and HT1080 cells were harvested by trypsin-EDTA and resuspended in Aldefluor assay buffer, containing ALDH substrate (BAAA, $1 \mu \mathrm{mol} / 1$ per $1 \times 10^{6}$ cells), and incubated for $30 \mathrm{~min}$ at $37^{\circ} \mathrm{C}$. As a negative control for each sample of cells, an aliquot was treated with $50 \mathrm{mmol} / \mathrm{l}$ diethylamino benzaldehyde (DEAB), an ALDH-specific inhibitor. The sorting gates were established using the Aldefluor-stained negative controls. Labeled cells were sorted with FACSaria and fractioned into ALDH-positive and negative cell populations. Both populations were then re-cultured, in monolayer conditions, and again harvested and examined using Aldefluor assay to determine whether both populations of ALDHpositive and negative cells could differentiate into original cell populations.

Sphere formation assay. Single cell suspensions dissociated with trypsin-EDTA of MG63 cells, at $\sim 70 \%$ confluence as well as both ALDH-positive and negative cells sorted by FACSaria were prepared for sphere formation assay. Cells were then inoculated into $\mathrm{N} 2 / 1 \%$ methylcellulose medium without serum at a density of $1 \times 10^{5}$ cells/well in ultra low attachment 6-well plates (Corning Inc., USA). N2 medium consists of 2X DMEM/F12 (Invitrogen, USA) supplemented with progesterone $(20 \mathrm{nM})$, putrescine $(100 \mu \mathrm{M})$, sodium selenite $(30 \mathrm{nM})$, transferrin $(25 \mu \mathrm{g} / \mathrm{ml})$, insulin $(20 \mu \mathrm{g} / \mathrm{ml})$ and human recombinant epidermal growth factor (EGF; $10 \mathrm{ng} / \mathrm{ml}$ ) and basic fibroblast growth factor (bFGF; $10 \mathrm{ng} / \mathrm{ml}$ ), mixed with an equal volume of $2 \%$ methylcellulose (22-24). All reagents were purchased from Sigma Biochemicals (USA). Fresh aliquots of EGF and bFGF were added every other day. After 10-14 days culture, sphere colonies were quantified by Olympus CK2 inverted phase contrast microscopy (Olympus Co., Japan). The secondary sphere formation ability through the repeating dissociation and re-culturing in anchorageindependent, serum-starved condition on ultra low attachment plates was also investigated.

Reverse transcription-polymerase chain reaction (RT-PCR). Total RNA was extracted from both monolayer cells, harvested by trypsin-EDTA, and sphere-forming cells using the FastPure RNA ${ }^{\circledR}$ kit (Takara Bio Inc., Japan) and firststrand cDNAs were synthesized from $500 \mathrm{ng}$ samples with PrimeScript $^{\circledR}$ RT-PCR kit (Takara Bio Inc.). All PCRs were performed using $0.5 \mu \mathrm{l}$ of a first-strand reaction mixture as templates. The primer sequences used for amplification were as follows: 5'-GCTGAGATGCCTCACACGGAG and
5'-TCTGTTTCTTGACTGGGACCTTGTC for Nanog; 5'TGGAGAAGGAGAAGCTGGAGCAAAA and 5'-GGCAG ATGGTCGTTTGGCTGAATA for Oct3/4; 5'-GGGTGGAG AAGGACACAGCGGTAA and 5'-GCCGACAATACTTTT CCGAATGC for STAT3; 5'-CCCCCCTGTGGTTACCTC TTC and 5'-TTCTCCCCCCTCCAGTTCG for Sox2; and 5'-TCACAGGATCAACAGAG and 5'-GTAGAATACCCA TGGTGTGC for ALDH1, respectively. The glyceraldehydes3-phosphate dehydrogenase (GAPDH) gene was used as an internal control to adjust the amounts of template using the primer pairs of 5'-CAGCCGAGCCACATCG and 5'-TG AGGCTGTTGTCATACTTCT. Aliquots $(10 \mu 1)$ of the amplification products were separated by electrophoresis using $1.5 \%$ agarose gels and visualized by ethidium bromide staining.

Efficacy of drugs on monolayer cells and spheres. MG63 cells were dissociated and inoculated into 96-well microtiter plates (Corning Inc., USA) at a concentration of 2000 cells/ $90 \mu \mathrm{l} /$ well, which were allowed to attach in DMEM/10\% FBS for $8 \mathrm{~h}$ at $37^{\circ} \mathrm{C}$. Cells were then exposed to $10 \mu \mathrm{l}$ of cisplatin (CDDP) or doxorubicin (DXR), at a final concentration of $10 \mu \mathrm{M}$ for $48 \mathrm{~h}$. Both drug-treated and non-treated cell viabilities were measured by MTS colorimetric assay, using CellTiter 96 Aqueous One Solution Cell Proliferation Assay reagent (Promega, USA), according to the manufacturer's instructions. For sphere-forming cells, MG63 cells were inoculated into 96-well ultra low attachment microplates (Corning Inc.) in N2/1\% methylcellulose media at a concentration of 5000 cells $/ 90 \mu 1 /$ well for 2 weeks to allow sphere formation, then treated with $10 \mu 1$ of cisplatin (CDDP) or doxorubicin (DXR) at a final concentration of $10 \mu \mathrm{M}$ for $48 \mathrm{~h}$ and cell viability was measured by MTS assay. The Student's t-test was carried out on five replicates of each experiment using STATA 8 (STATA Corp., USA).

\section{Results}

Separation and differentiation of the ALDH-positive and negative populations from human sarcoma cell lines. We firstly assessed the presence and size of the cell populations with ALDH enzymatic activity in MG63 human osteosarcoma and HT1080 fibrosarcoma cell lines by Aldefluor assay. Results showed that both cell lines contained the populations of cells exhibiting ALDH activity, with frequencies of $11 \%$ in MG63 cells and 9\% in HT1080 cells (Fig. 1B and D), compared to their control with ALDH inhibitor DEAB with frequencies of 0.75 and $0.77 \%$, respectively (Fig. 1A and C). Following this, we separated the high and low ALDH activity populations in the MG63 cell line using FACSaria cell sorting. The high and low ALDH activity populations were collected at rates of 9 and $6 \%$, respectively (Fig. 1E). ALDH1 mRNA expression levels were correlated with cell sorting by ALDH activity (Fig. 1F). After sorting, both high and low ALDH activity cells were cultured in monolayer conditions with DMEM-10\% FBS. These were then harvested and analyzed by Aldefluor assay at $\sim 70 \%$ confluence. Both cell populations showed the same pattern of ALDH positive and negative activity as the parental MG63 cells (Fig. 1G; data not shown for low ALDH 
A

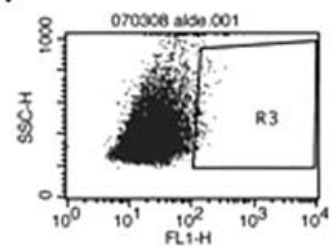

MG63 control; R3: $0.75 \%$

$C$

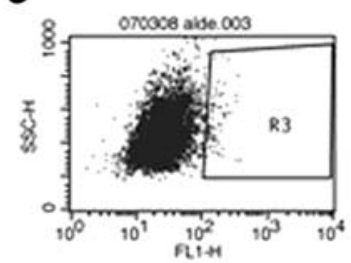

HT1080 control: R3 : $0.77 \%$

E

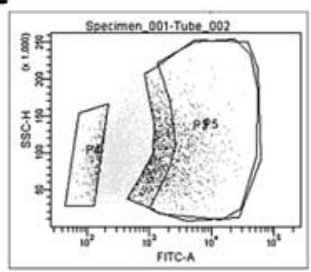

ALDH1 negative (p4): $6.3 \%$ ALDH1 positive ( $\mathrm{p} 5)$ : $9 \%$

$\mathbf{F}$

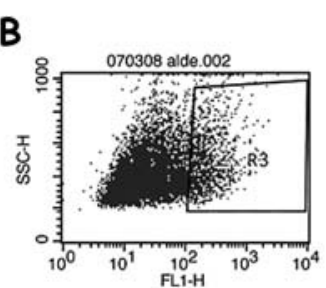

MG63: R3: $11.59 \%$

$D$

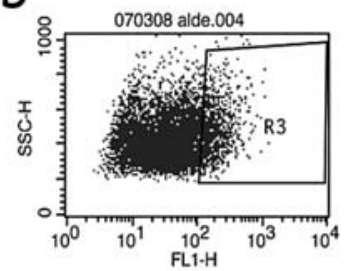

HT1080: R3: $9.40 \%$

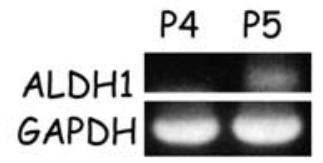

mRNA expression of ALDH1

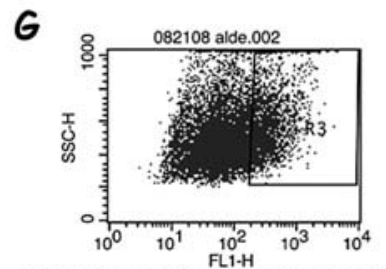

ALDH1 positive cells $(R 3): 22 \%$

Figure 1. (A) FACS analysis of MG63 with ALDH inhibitor DEAB as a control. (B) Aldefluor assay showing $11 \%$ of the cell population in human osteosarcoma MG63 express high ALDH1 activity. (C) FACS analysis of HT1080 with ALDH inhibitor DEAB as a control. (D) Nine percent of the human fibrosarcoma HT1080 cell population express high ALDH1 activity. (E) Cell sorting by ALDH1 activity isolated 6\% of the low ALDH activity population and 9\% of the high ALDH activity population from the MG63 cell line. (F) Expression of ALDH1 mRNA correlates with cell sorting isolation. (G) Subculture of high ALDH population from MG63 in monolayer conditions show that cells could differentiate from high ALDH to low ALDH activity population and the ratio of high ALDH population was relatively high (22\%) compared with parental MG63 (11\%).

activity population), suggesting that both populations have the ability for differentiation.

Formation and stem-like characterization of sarcospheres. Spherical colonies were formed over several days after culturing in anchorage-independent, serum-starved conditions (Fig. 2a). The frequencies of sphere formation were $\sim 0.25$ and $\sim 0.78 \%$ for MG63 and HT1080 cells, respectively. ALDH1-positive cells showed high frequency of sphere formation in contrast to ALDH1-negative cells. Dissociated sphere cells were capable growing in monolayer conditions similarly to the parental cells, and repeatedly formed spheres by re-culturing in anchorage-independent, serum-starved conditions, as the first generation of spheres (Fig. 2b). RT-
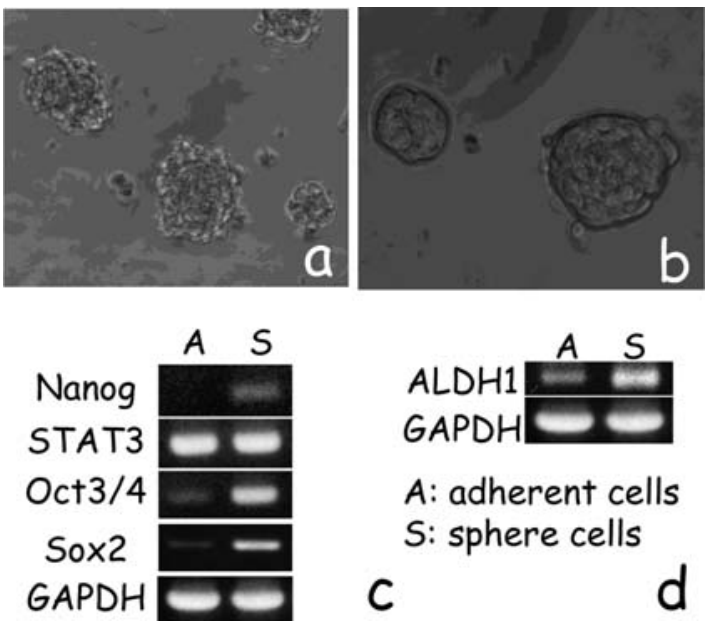

Figure 2. (a) Primary sphere formation from MG63 osteosarcoma cells in anchorage-independent, serum-free conditions and (b) dissociated MG63 primary sphere cells showed a capacity for secondary sphere formation. (c) mRNA expression of stem cell-related genes show a significant increase of Nanog, Oct3/4 and Sox 2 genes in sphere cells from MG63 and (d) a significant increase in ALDH1 expression in sphere-forming cells.

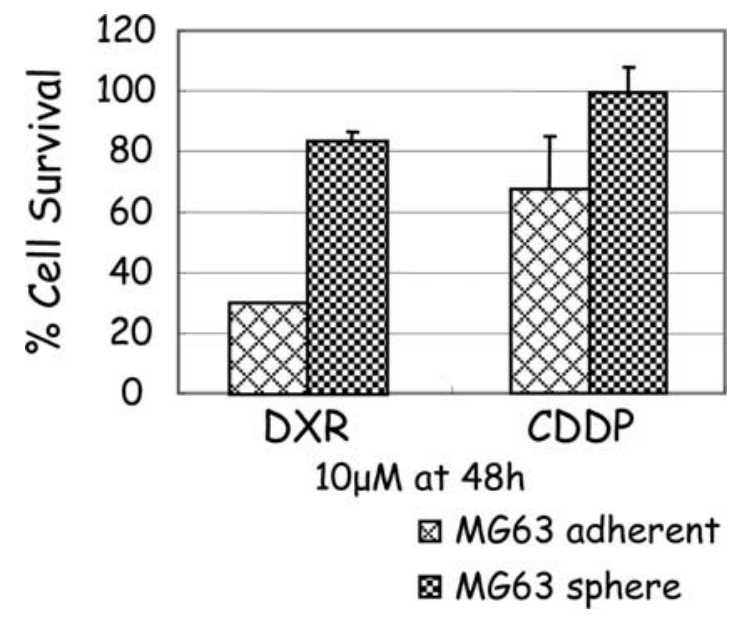

Figure 3. Sphere-forming stem-like cells showed statistically significant increased drug resistance, compared with monolayer, adherent cells $(\mathrm{p}<0.05)$.

PCR revealed that cells present in the spheres strongly express the stem cell-related genes, Nanog, Oct $3 / 4$, Stat 3 and Sox 2, compared with monolayer cells (Fig. 2c). This indicates that sphere-forming cells have stem-like properties. Furthermore, ALDH1 mRNA expression was also increased in sphere-forming cells compared to monolayer cells (Fig. $2 \mathrm{~d}$ ). The frequencies of sphere-forming populations were very small compared to the ratios of the high ALDH activity populations in total cell population, suggesting that the high ALDH activity populations contain stem-like populations, but not entirely represent them in these cell lines.

Drug resistance of spheres from human osteosaroma cell line. Both CDDP and DXR inhibited the growth of MG63 up to 68 and $30 \%$, respectively at a concentration of $10 \mu \mathrm{M}$ for $48 \mathrm{~h}$ treatment. In contrast, sphere-forming cells from MG63 showed statistically significant strong resistance to both agents (Fig. 3), suggesting stem-like sphere-forming cells exhibiting 


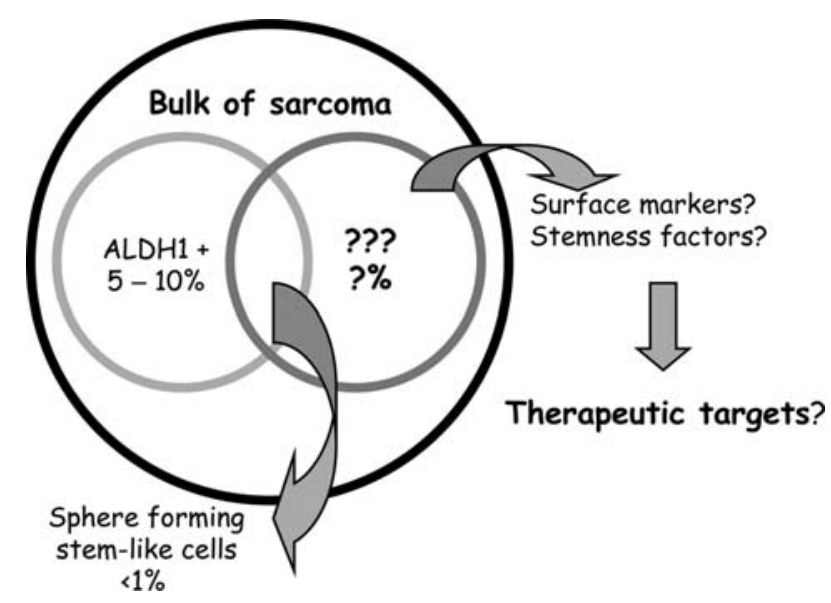

Figure 4. Future direction of sarcoma treatment strategies may focus on defining sarcoma stem cells by surface or true stemness markers, thereby targeting them to eliminate sarcoma-initiating cells.

high ALDH activity are resistant to CDDP and DXR, both of which are key drugs for sarcoma treatment.

\section{Discussion}

Cancer stem cell hypothesis depicts that tumors originate in tissue stem cells or progenitor cells through deregulation of their stem cell properties. Furthermore, it is also likely that cancer stem cells have a phenotype defined by their cell of origin (stem or early progenitor cells) and retain the stem cell properties of self-renewal, capacity to differentiate and drug resistance. These cells may initiate tumor growth and give rise to a heterogeneous population of cancer cells. Recently numerous investigations have demonstrated the presence of stem-like cell populations in various types of cancers (4-11), some of which were defined their populations with cell surface markers. However, in certain cancers, especially sarcomas, stem-like cells cannot be identified by surface markers as there are no known markers for their normal counterpart, e.g., mesenchymal stem cells. In early investigations, sidepopulation (SP) were a distinct subset of cells defined by Hoechst exclusion and considered to be an enriched source of primitive and undifferentiated cells (25). The SP most likely contains stem cells, but not every SP cell is a stem cell and some stem cells may not be included in the SP (26). With the growing need for new markers to identify and isolate stem cells, ALDH1 activity has been proposed as a promising candidate. It functions as a detoxifying enzyme and has been proposed as a common marker for both normal and malignant stem and progenitor cells $(17,19,27)$. ALDH1 activity has been employed successfully as a stem cell marker in retinoblastoma, prostate, pancreas and breast cancer (20,28-30). The current study demonstrated that both human osteosarcoma MG63 and human fibrosarcoma HT1080 contained subpopulations with high ALDH activity, approximately $10 \%$ of the total cell population, as determined by Aldefluor assay. After cell sorting, the population with high ALDH activity from the MG63 cell line showed a capacity for differentiation, giving rise to both high and low ALDH activity populations after re-culturing in monolayer conditions. More interestingly, the low ALDH activity population also gave rise to both high and low ALDH populations. Although there were possibilities of contaminants of ALDH1-positive cells in the sorting process, or that the expression of ALDH1 may be turned on and off during differentiation, one possible explanation would be the certain reprogramming pathways existing in these populations that can induce dedifferentiation from the differentiated low ALDH activity population to the more undifferentiated high ALDH activity population, like induced pluripotent stem (iPS) cells, which can be induced from the certain differentiated cell types by the introduction of certain genes $(31,32)$. Otherwise, these cells may not be hierarchically organized.

Sphere-forming populations showed an increased expression of stem cell-related genes and a capacity for self renewal, thereby exhibiting stem-like properties. ALDH1 mRNA expression was also increased in these stem-like sphereforming cells. However, frequencies of sphere-forming populations were much smaller compared with the ratio of the populations with high ALDH1 activity in total cell population. Moreover, not all high ALDH activity cells formed stem-like spheres (data not shown), which has also been previously described for the SP cells (26), suggesting that the high ALDH activity population is not completely composed of stem-like cells. These results suggest that although high ALDH activity is present in stem-like populations, it is not sufficient enough to isolate these cells as a single marker. Further study is required to elucidate a definitive maker for sarcoma stem cells, such as specific surface markers (Fig. 4).

The mechanism by which drug resistance occurs in stem cells or cancer stem cells has not yet been fully elucidated. Possible mechanisms have been proposed which include the following: i) activation and/or overexpression of cell membrane drug efflux transporters; ii) altered expression of detoxifying enzymes; iii) efficient DNA repair and defective apoptosis regulatory proteins; and vi) 'niche' protection of target cells. The presence of cancer stem cells was originally demonstrated through the presence of side-populations by Hoechst dye efflux assay (25), which fundamentally questions drug resistance in cancer stem cells by the activation of drug efflux pumps. The current study suggests that increased expression of the detoxifying enzyme, ALDH1, could be one of the possible mechanisms responsible for the development of drug resistance in cancer stem cells derived from human sarcomas. Our previous study demonstrated that the expression of DNA repair enzymes, MLH1 and MSH2, were increased in spherical stem-like cells from human sarcoma cell lines, suggesting that efficient DNA repair could contribute to drug resistance in these cells (24). Together, these results indicate that the presence of stem-like cells with increased ALDH1 expression and efficient DNA repair could be one of the possible contributors to the development of drug resistance in sarcomas. The results from these studies may provide future directions for further investigation in targeting of sarcoma stem cells.

In conclusion, the presence of stem-like cells could be a possible pathogenesis for drug resistance in sarcomas. Further study will be required to define the sarcoma stem cells and the mechanisms of drug resistance, but future treatment strategy would be changed along with the develop- 
ments of this filed in conjunction with mesenchymal stem cell biology.

\section{Acknowledgements}

This study was supported by a Grant-in-Aid for Scientific Research (No. 20591765 to K.H.) from Ministry of Education, Culture, Sports, Science and Technology, Japan.

\section{References}

1. Sell S: Stem cell origin cancer and differentiation therapy. Crit Rev Oncol Hematol 51: 1-28, 2004.

2. Reya T, Morison SJ, Clarke MF and Weissman IL: Stem cells, cancer and cancer stem cells. Nature 414: 105-111, 2001.

3. Molofsky AV, Pardel R and Morrison SJ: Diverse mechanisms regulate stem cell self-renewal. Curr Opin Cell Biol 16: 700-707, 2004.

4. Bonnet D and Dick JE: Human acute myeloid leukemia is organized as a hierarchy that originates from a primitive hematopoietic cell. Nat Med 3: 730-737, 1997.

5. Al-Haji M, Wicha MS, Benito-Hernandez A, Morrison SJ and Clarke MF: Prospective identification of tumorigenic breast cancer cells. Proc Natl Acad Sci USA 100: 3983-3988, 2003.

6. Singh SK, Hawkins C, Clarke ID, et al: Identification of human brain tumor initiating cells. Nature 43: 396-401, 2004.

7. Collins AT, Berry PA, Hyde C, Stower MJ and Maitland NJ: Prospective identification of tumorigenic prostate cancer stem cells. Cancer Res 65: 10946-10951, 2005.

8. Fang D, Nguyen TK, Leishear K, et al: A tumorigenic subpopulation with stem cell properties in melanomas. Cancer Res 65: 9328-9337, 2005.

9. O'Brien CA, Pollett A, Gallinger S and Dick JE: A human colon cancer cell capable of initiating tumor growth in immunodeficient mice. Nature 445: 106-110, 2006.

10. Li C, Heidt DG, Dalerba P, et al: Identification of pancreatic cancer stem cells. Cancer Res 67: 1030-1037, 2007.

11. Prince ME, Sivanandan R, Kaczorowski A, et al: Identification of a subpopulation of cells with cancer stem cell properties in head and neck squamous cell carcinoma. Proc Natl Acad Sci USA 104: 973-978, 2007.

12. Taussig DC, Pearce DJ, Simpson C, et al: Hematopoietic stem cells express multiple myeloid markers: implications for the origin and targeted therapy of acute myeloid leukemia. Blood 106: 4086-4092, 2005.

13. Russo JE and Hilton J: Characterization of cytosolic aldehyde dehydrogenase from cyclophosphamide resistant L1210 cells. Cancer Res 48: 2963-2968, 1988.

14. Magni M, Shammah S, Schiro R, Mellado W, Dalla-Favera R and Gianni AM: Induction of cyclophosphamide-resistance by aldehyde-dehydrogenase gene transfer. Blood 87: 1097-1103, 1996.

15. Chute JP, Muramoto GG, Whitesides J, et al: Inhibition of aldehyde dehydrogenase and retinoid signaling induces the expansion of human hematopoietic stem cells. Proc Natl Acad Sci USA 103: 11707-11712, 2006.

16. Sahovic EA, Colvin M, Hilton J and Ogawa M: Role for aldehyde dehydrogenase in survival of progenitors for murine blast cell colonies after treatment with 4-hydroperoxycyclophosphamide in vitro. Cancer Res 48: 1223-1226, 1988.
17. Storms RW, Trujillo AP, Springer JB, et al: Isolation of primitive human hematopoietic progenitors on the basis of aldehyde dehydrogenase activity. Proc Natl Acad Sci USA 96: 9118-9123, 1996.

18. Sladek NE, Kollander R, Sreerama L and Kiang DT: Cellular levels of aldehyde dehydrogenases (ALDH1A1 and ALDH3A1) as predictors of therapeutic response to cyclophosphamidebased chemotherapy of breast cancer: a retrospective study. Rational individualization of oxazaphosphorine-based cancer chemotherapeutic regimens. Cancer Chemother Pharmacol 49: 309-321, 2002.

19. Pearce DJ, Taussig D, Simpson C, et al: Characterization of cells with a high aldehyde dehydrogenase activity from cord blood and acute myeloid leukemia samples. Stem Cells 23: 752-760, 2005.

20. Ginestier C, Hur MH, Charafe-Jauffret E, et al: ALDH1 is a marker of normal and malignant human mammary stem cells and a predictor of poor clinical outcome. Cell Stem Cell 1: 555-567, 2007.

21. Jones RJ, Barber JP, Vala MS, et al: Assessment of aldehyde dehydrogenase in viable cells. Blood 85: 2742-2746, 1995.

22. Gibbs CP, Kukekov VG, Reith JD, et al: Stem-like cells in bone sarcomas: implications for tumorigenesis. Neoplasia 7: 967-976, 2005.

23. Fujii H, Honoki K, Tsujiuchi T, et al: Reduced expression of INK4a/ARF genes in stem-like sphere cells from rat sarcomas. Biochem Biophys Res Commun 362: 773-778, 2007.

24. Fujii H, Honoki K, Tsujiuchi T, Kido A, Yoshitani K and Takakura Y: Sphere-forming stem-like cell populations with drug resistance in human sarcoma cell lines. Int J Oncol 34: 1381-1386, 2009.

25. Hadnagy A, Gaboury L, Beauliue R and Balicki D: SP analysis may be used to identify cancer stem cell populations. Exp Cell Res 312: 3701-3710, 2006.

26. Smalley MJ and Clarke RB: The mammary gland "side population': a putative stem/progenitor cell marker? J Mammary Gland Biol Neoplasia 10: 37-47, 2005.

27. Hess DA, Meyerrose TE, Wirthlin L, et al: Functional characterization of highly purified human hematopoietic repopulating cells isolated according to aldehyde dehydrogenase activity. Blood 104: 1648-1655, 2004.

28. Armstrong L, Stojkovic M, Dimmick I, et al: Phenotypic characterization of murine primitive hematopoietic progeniotor cells isolated on basis of aldehyde dehydrogenase activity. Stem Cells 22: 1142-1151, 2004.

29. Siegel GM, Campbell LM, Narayan M and GonzalezFernandez F: Cancer stem cell characteristics in retinoblastoma. Mol Vis 12: 729-737, 2005.

30. Kim H, Lapointe J, Kaygusuz G, et al: The retinoic acid synthesis gene ALDH1a2 is a candidate tumor suppressor in prostate cancer. Cancer Res 65: 8118-8124, 2005.

31. Takahashi K and Yamanaka S: Induction of pluripotent stem cells from mouse embryonic and adult fibroblast cultures by defined factors. Cell 126: 663-676, 2006.

32. Takahashi K, Tanabe K, Ohnuki M, et al: Induction of pluripotent stem cells from adult human fibroblasts by defined factors. Cell 131: 861-872, 2007. 